\title{
Colonização de substrato artificial pelo mexilhão dourado, Limnoperna fortunei (Dunker, 1857) (Bivalvia, Mytiloida, Mytilidae), no Delta do Rio Jacuí (RS, Brasil)
}

\author{
Maria Cristina Dreher Mansur ${ }^{1 *}$ \\ Daniel Pereira ${ }^{1}$ \\ Cíntia Pinheiro dos Santos ${ }^{1}$ \\ Paulo Eduardo Aydos Bergonci ${ }^{1}$ \\ Bruno Mitidiero Thormann² \\ Alice Michiyo Takeda ${ }^{3}$ \\ ${ }^{1}$ Laboratório de Malacologia, Museu de Ciências e Tecnologia \\ Pontifícia Universidade Católica do Rio Grande do Sul \\ Av. Ipiranga 6681, prédio 40, CEP 90619-900, Porto Alegre - RS, Brasil \\ ${ }^{2} \mathrm{PPG}$ em Aqüicultura, Universidade Federal de Santa Catarina, Florianópolis - SC, Brasil \\ ${ }^{3}$ Universidade Estadual de Maringá, Departamento de Biologia, UEM/Nupelia \\ Maringá - PR, Brasil \\ *Autora para correspondência \\ momansur@terra.com.br
}

\section{Resumo}

Com o objetivo de avaliar a colonização de substrato artificial de madeira (em forma de X) pelo mexilhão dourado, Limnoperna fortunei (Dunker, 1857), como subsídio aos programas de monitoramento da invasora, foram realizados experimentos no delta do rio Jacuí (RS). Em dezembro de 2005 foram expostas (por três meses) três réplicas do substrato, em diferentes profundidades $(1,1,5$ e $2 \mathrm{~m})$, em duas estações de coleta (total de 18 réplicas), no delta do rio Jacuí, no município de Porto Alegre: no Canal do Jacuí (CJ) e na doca do Cais do Porto (PO). Os substratos foram secos em forno de Pasteur $\left(60^{\circ} \mathrm{C}\right)$. Os indivíduos adultos $(>0,5 \mathrm{~mm})$ incrustados nas diferentes posições no substrato (superior, inferior, lateral 1 e 2) foram removidos e quantificados sob o estereomicroscópio. A densidade média (ind. - $^{-2}$ ) e o erro padrão nas estações de coleta foram: CJ, 309,19 \pm 30,7 e PO, 39,4 \pm 6,0. O teste de Kruskal-Wallis $(\alpha=0,05)$ não demonstrou diferenças significativas entre profundidades $(p=0,715) \mathrm{e}$ posições do substrato $(p=0,617)$, mas o Teste $U$ demonstrou diferenças $(p<0,0001)$ entre estações de coleta. As densidades médias de adultos verificadas neste estudo foram superiores aos valores encontrados para o mesmo substrato, no rio Paraguai, e inferiores aos valores verificados no rio Paraná.

Unitermos: substrato artificial, colonização, invasoras, delta interior subtropical 


\section{Abstract}

Colonization of artificial substrate by golden mussel, Limnoperna fortunei (Dunker, 1857), in the Jacuí River Delta, Rio Grande do Sul State, Brazil. In order to test a colonization method for Limnoperna fortunei, experiments were carried out in the Jacuí River Delta (Rio Grande do Sul State), using wood substrate (X form). The sampling localities are in the Jacuí River Delta: Jacuí Canal (CJ) and Port Docks (PO). In December (2005), three replicas of substrate were exposed at different depth levels $(1 ; 1.5$ and $2 \mathrm{~m})$ in each sampling locality (total of 18 replicas). In March, all substrates were collected from the water and transported to the laboratory. The substrates were dehumidified in a Pasteur oven $\left(60^{\circ} \mathrm{C}\right)$. The individual adults $(>0.5 \mathrm{~mm})$ incrusted on different sides of the substrate (upperside, underside, lateral sides 1 and 2) were removed and quantified under a stereoscopic microscope. The average densities (ind. $\mathrm{m}^{-2}$ ) and standard errors of golden mussel adults were: CJ, $309.19 \pm 30.70$ and PO, $39.40 \pm 6.00$. The Kruskal-Wallis test $(\alpha=0.05)$ did not present any significant differences between the depth levels $(\mathrm{p}=0.715)$ or substrate sides $(\mathrm{p}=0.617)$. However, the $U$ Test presented differences $(p<0.0001)$ between sampling localities. The average densities of adult individuals verified in this study were situated between extreme values recorded earlier for wood substrate colonization, i. e. they were higher than those obtained for the Paraguay River and lower than those obtained for the Paraná River.

Key words: artificial substrate, colonization, invasors, subtropical inner delta

\section{Introdução}

O mexilhão dourado é um bivalve invasor de origem asiática que foi introduzido no Brasil inicialmente na bacia do lago Guaíba, em Porto Alegre, RS (Mansur et al., 1999 e 2003). Com o passar dos anos, dispersou-se pelos afluentes do lago Guaíba e pela laguna dos Patos causando alterações na paisagem, fauna e flora aquática, acarretando prejuízos econômicos aos sistemas de captação e tratamento de água por meio da formação de macroaglomerados compactos (Mansur et al., 2003 e 2004; Darrigran e Mansur, 2006).

Limnoperna fortunei produz fios de bisso que possibilitam a macroaglomeração sobre substratos duros (Mansur e Pereira, 2006). Os substratos artificiais são ferramentas amplamente utilizadas no monitoramento de macroaglomerados de mexilhão dourado. O substrato artificial cerâmico foi amplamente utilizado no Rio Grande do Sul (Mansur et al., 2003; Santos, 2004; Mansur, 2007; Terra et al., 2007; Santos et al., 2008), especialmente na bacia do lago Guaíba. Em um reservatório em Hong Kong (China), Morton (1982) avaliou a colonização de substratos artificiais (abestos) pelo mexilhão dourado. Posteriormente, foram realizadas algumas adaptações do substrato utilizado em Hong Kong por Boltovskoy e Cataldo (1999), para monitorarem populações de mexilhão dourado no rio Paraná; Fontes et al. (2002), no lago de Itaipú Binacional;
Darrigran et al. (2007) no lago de Yacyretá (ArgentinaParaguay) e por Oliveira et al. (2006), na bacia do rio Paraguai.

O presente trabalho objetivou avaliar a colonização de substrato artificial de madeira (em forma de X) pelo mexilhão dourado, Limnoperna fortunei (Dunker, 1857), em diferentes profundidades e posicionamentos no substrato, com intuito de fornecer subsídios para a interpretação da formação de macroaglomerados em substratos artificiais, em programas de monitoramento da invasora na referida área de estudo.

\section{Material e Métodos}

A área (Figura 1) está situada no delta do rio Jacuí, na unidade fisiográfica da Depressão Central do Estado do Rio Grande do Sul, na região metropolitana de Porto Alegre.

O rio Jacuí deságua no lago Guaíba por meio de um delta formado por uma série de distributários, dentre os quais se destaca o Canal do Jacuí (profundidade média: $8 \mathrm{~m})$. No Cais do Porto, junto à área urbana de Porto Alegre, a paisagem marginal foi significativamente alterada por meio de um aterro. Atualmente, neste local se verificam margens íngremes (paredes concretadas) e águas profundas $(5 \mathrm{~m})$. As estações de coleta situam-se no delta do rio Jacuí: na margem direita do Canal do 
Jacuí, junto a Ilha da Pintada (CJ), e no Cais do Porto (PO), junto à doca do Corpo de Bombeiros.

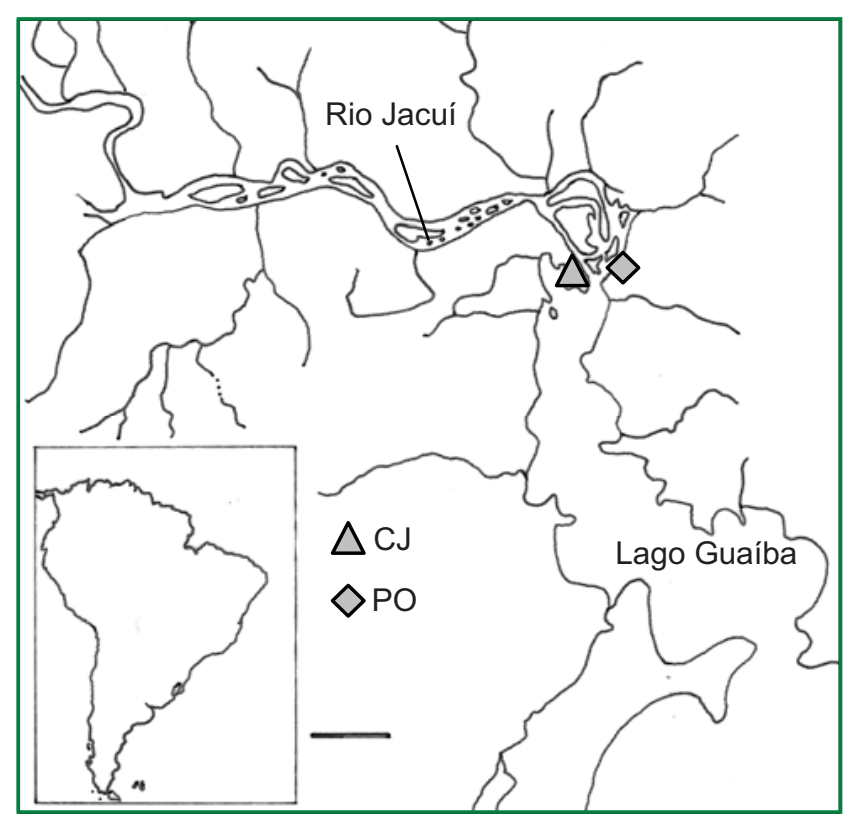

FIGURA 1: Estações de coleta no Canal do Jacuí, na Ilha da Pintada (CJ), delta do rio Jacuí e no Cais do Porto (PO), no lago Guaíba (RS, Brasil) (Barra $5 \mathrm{~km}$ ).
O período dezembro de 2005 a março de 2006 foi determinado para a realização dos experimentos de exposição de substratos artificiais, época de maior produção larval e recrutamento, a partir da análise de dados históricos das densidades de larvas no baixo rio Paraná (Boltovskoy e Cataldo, 1999), no rio Paraná superior (Darrigran et al., 2007) e no lago Guaíba (Santos, 2004). O tempo de exposição (três meses) seria então o mínimo necessário para a colonização (recrutas) e desenvolvimento dos espécimes (adultos) (Boltovskoy e Cataldo, 1999).

Em dezembro (2005) foram expostos três conjuntos $\left(\mathrm{A}_{\mathrm{n}}, \mathrm{B}_{\mathrm{n}}\right.$ e $\left.\mathrm{C}_{\mathrm{n}}\right)$ de três réplicas $(\mathrm{n}=1,2$ e 3$)$ de substrato "X" de madeira em cada uma das estações de coleta (CJ e PO). As três réplicas de cada conjunto foram interligadas por cordas de aproximadamente $0,5 \mathrm{~m}$. A corda fixada a última réplica continha um peso (tijolo cerâmico) servindo como âncora com a finalidade de manter o conjunto de réplicas em posição vertical (Figura 2). Sendo assim, as três réplicas em cada conjunto foram posicionadas às profundidades aproximadas de 1,$0 ; 1,5$ e $2,0 \mathrm{~m}$, respectivamente.
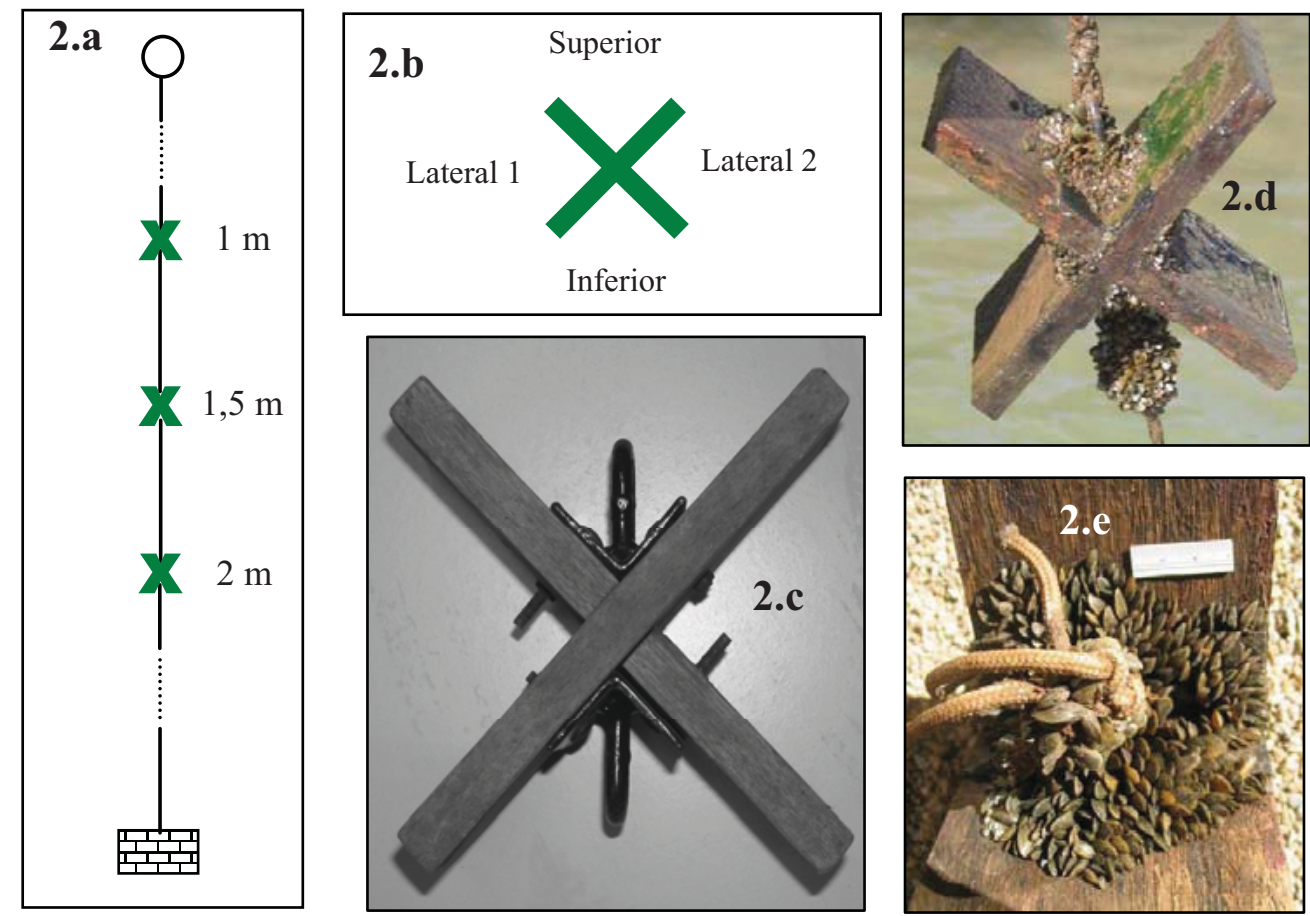

FIGURA 2: Substrato artificial de madeira em forma de X: 2a, substratos suspensos por cordas em diferentes profundidades, ancorados no sedimento por um tijolo cerâmico; $2 \mathrm{~b}$, terminologia do substrato; $2 \mathrm{c}$, substrato sem incrustação (área: $0,072 \mathrm{~m}^{2}$ ); $2 \mathrm{~d}$, visão geral do substrato incrustado e 2e, visão da superfície superior do substrato com incrustações. 
No mesmo período foram realizadas amostragens mensais de larvas (30L) em ambas estações de coleta com rede de plâncton $(30 \mu \mathrm{m})$ de acordo com Santos et al. (2005). O acompanhamento das densidades larvais foi utilizado como um indicador de recrutamento potencial. As larvas foram quantificadas (ind. $\mathrm{m}^{-3}$ ) de acordo com Santos et al. (2005).

Todas as réplicas de substrato de madeira foram desumidificadas em forno de Pasteur $\left(60^{\circ} \mathrm{C}\right)$. Aglomerados formados nas posições superior, lateral 1 e 2, e inferior (Figura 2) do substrato, foram removidos individualmente e os espécimes adultos $(\geq 0,5 \mathrm{~mm}$ de comprimento) quantificados, obtendo-se assim a abundância. Com base na área amostral do substrato artificial $\left(0,0722 \mathrm{~m}^{2}\right)$ também foram calculadas as densidades médias expressas em $\mathrm{m}^{2}$, para cada estação de coleta.

As abundâncias de indivíduos adultos dos aglomerados nas diferentes posições do substrato foram comparadas por meio do teste de Kruskal-Wallis $(\alpha=0,05)$. O mesmo procedimento foi utilizado para comparar as abundâncias nas diferentes profundidades. A comparação de abundâncias das estações de coleta foi realizada por meio do Teste $U(\alpha=0,05)$. A escolha dos testes não-paramétricos ocorreu após a verificação da não normalidade dos dados por meio do teste KolmogorovSmirnov para uma amostra $\left(\mathrm{H}_{0}\right.$ : dados seguem distribuição normal), com nível de significância de 95\%, de acordo com Zar (1999). A ferramenta utilizada para a obtenção do teste de Kruskal-Wallis, do Teste U e o de Kolmogorov-Smirnov, foi o software SPSS 11.5.

\section{Resultados e Discussão}

Densidade média mensal de larvas. No período do experimento a densidade média (ind. $\mathrm{m}^{-3}$ ), o erro padrão, a densidade mínima e máxima de larvas no $\mathrm{CJ}$ foram respectivamente, $7208,3 \pm 2026,1(1500,0-11066,6) \mathrm{e}$ no PO, 2000, $0 \pm 751,7$ (700,0 - 3866,7). O CJ apresentou as maiores densidades médias de larvas, com valor máximo no mês de janeiro. Foram verificadas diferenças significativas $(\mathrm{p}=0,08)$ dentre as densidades larvais do $\mathrm{CJ}$ e PO, quando considerado $\alpha<0,1$.
Abundância versus posição no substrato. O número amostral, o valor médio, o erro padrão e os valores mínimos e máximos verificados nas quatro posições foram (Figura 3) respectivamente: inferior, $n$ $=18,251,5 \pm 61,2(18,00-851,00)$; lateral $1, \mathrm{n}=18$, $149,4 \pm 33,0(2,00-510,00)$; lateral $2, \mathrm{n}=18,145,4$ $\pm 34,7(8,00-546,00)$; e superior, $\mathrm{n}=18,150,8 \pm 42,5$ $(7,00-669,00)$.

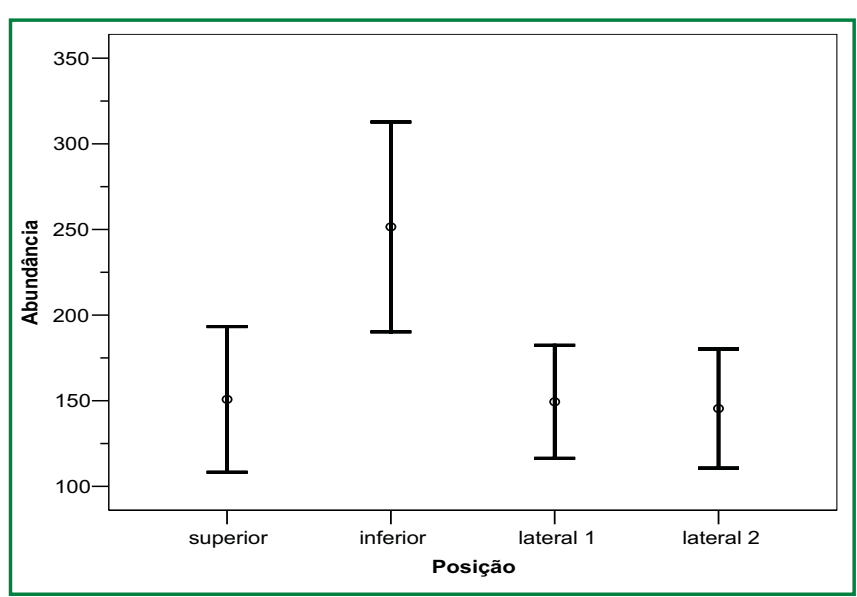

FIGURA 3: Abundância (indivíduos adultos) de Limnoperna fortunei (Dunker, 1857), com respectivo erro padrão nas diferentes superfícies (S, superior; L1, lateral $1 \mathrm{e}$ L2, 2; I, inferior), em ambas estações de coleta (CJ e PO), no delta do rio Jacuí, Porto Alegre, RS, Brasil.

O teste de Kruskal-Wallis $(\mathrm{p}=0,617)$ não demonstrou diferenças significativas entre as diferentes posições. Avaliando a colonização de substrato de madeira (placas), Marques-Silva et al. (2006) verificaram que as densidades do bivalve bissado Mytella falcata (d'Orbigny, 1846) foram menos elevadas na posição superior do que na posição inferior do substrato. $\mathrm{O}$ autor atribuiu esta preferência ao possível acúmulo de silte e ao risco de dessecação na superfície superior da placa formando uma barreira ao assentamento. No presente experimento não foi constatado acúmulo de silte sobre a parte superior do substrato, muito menos exposição ao ar acarretando em dessecação.

Abundância versus profundidade. O número amostral, o valor médio, o erro padrão e os valores mínimos e máximos verificados nas três profundidades foram (Figura 4) respectivamente: $1 \mathrm{~m}, \mathrm{n}=24,183,91 \pm$ $40,61(10,00-851,00) ; 1,5 \mathrm{~m}, \mathrm{n}=24,151,7 \pm 32,6(2,00$ $-521,00)$ e $2 \mathrm{~m}, \mathrm{n}=24,187,2 \pm 43,2(7,00-669,00)$. 
O teste de Kruskal-Wallis ( $\mathrm{p}=0,715)$ não demonstrou diferenças significativas entre réplicas expostas em diferentes profundidades. Fontes et al. (2002) também não verificaram diferenças significativas em substratos de madeira $(\mathrm{X})$ submersos em profundidades de 1,5 e $3,0 \mathrm{~m}$. No entanto, na linha da superfície da água, onde permaneceu a bóia, na qual os substratos foram suspensos, os autores verificaram ausência de mexilhão dourado na maioria das amostras.

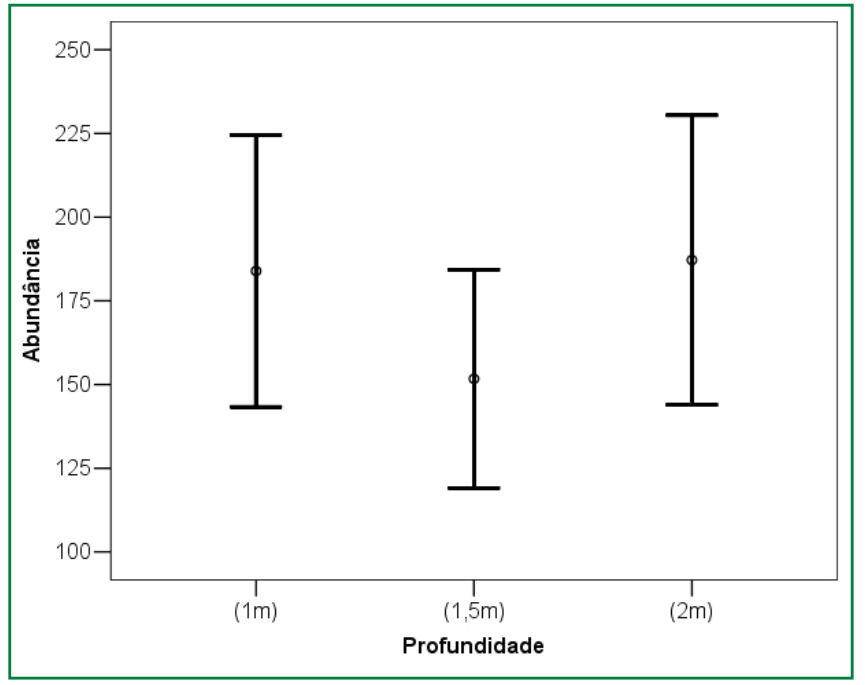

FIGURA 4: Abundância (indivíduos adultos) de Limnoperna fortunei (Dunker, 1857), com respectivo erro padrão nas diferentes profundidades (aproximadamente 1; 1,5 e $2 \mathrm{~m}$ ), em ambas estações de coleta (CJ e PO), no delta do rio Jacuí, Porto Alegre, RS, Brasil.

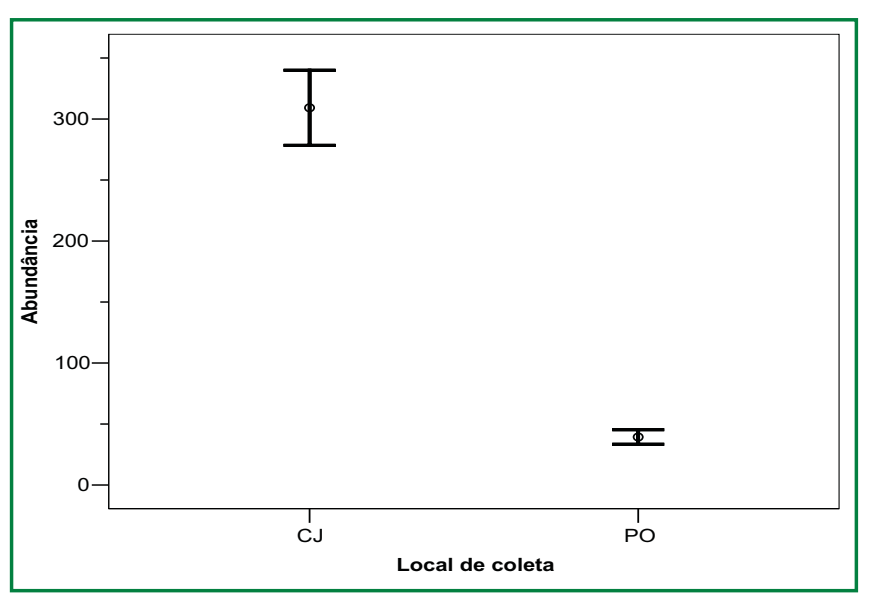

FIGURA 5: Abundância de espécimes adultos de mexilhão dourado, com respectivo erro padrão nas estações de coleta (CJ e PO), no delta do rio Jacuí, Porto Alegre, RS, Brasil.
Abundância versus estação de coleta. O número amostral, o valor médio, o erro padrão e os valores mínimos e máximos verificados nas duas estações de coleta foram (Figura 5) respectivamente: CJ, $\mathrm{n}=36$, $309,19 \pm 30,7(27,00-851,00)$ e PO, $n=36,39,4 \pm 6,0$ $(2,00-143,00)$.

O teste do Teste $U$ demonstrou diferenças significativas $(p<0,0001)$ entre réplicas expostas nas duas estações de coleta. Mansur (dados inéditos) também verificou densidades médias elevadas de indivíduos adultos de Limnoperna fortunei incrustados sobre substrato cerâmico e garrafa PET no $\mathrm{CJ}$ e densidades reduzidas no PO. As diferenças entre as densidades populacionais nestas estações de coleta, provavelmente estiveram relacionadas à qualidade da água. Faria e Lersch (1998) e Bendati et al. (2000) realizaram monitoramentos de longo período no delta do rio Jacuí e no lago Guaíba respectivamente, constatando águas de melhor qualidade (Classe 2) no CJ e águas de pior qualidade (Classe 4, de acordo com a resolução $\mathrm{n}^{\circ} 20$ do CONAMA (1986)) no PO.

Densidade média no substrato de madeira (X). $\mathrm{O}$ número amostral, o valor médio, o erro padrão e os valores mínimos e máximos verificados foram respectivamente: $\mathrm{CJ}, \mathrm{n}=9,17177,4 \pm 1249,3$ (11972,2 $-21763,9)$ e PO, $\mathrm{n}=9,2186,7 \pm 480,1(708,3-4930,6)$. As densidades verificadas no presente estudo foram inferiores as densidades $\left(0-70.000\right.$ ind. $\left.\mathrm{m}^{-2}\right)$ observadas por Fontes et al. (2002), no lago de Itaipu e superiores as densidades $\left(0-523,8\right.$ ind. $\left.\mathrm{m}^{-2}\right)$ constatadas por Oliveira et al. (2006), no rio Paraguai. No lago de Itaipu, a escassez de substratos naturais apropriados, pode ter acarretado na colonização mais intensa do substrato artificial. Neste lago o fundo é arenoso. No rio Paraguai, as baixas densidades verificadas por Oliveira et al. (2006) podem ser atribuídas à recente invasão de Limnoperna fortunei na época do estudo.

O substrato testado apresentou colonização uniforme em toda a sua superfície e nas diferentes profundidades. No entanto, comparações num maior gradiente de profundidades em rios, lagos ou reservatórios poderiam evidenciar padrões diferenciados dos constatados neste estudo. O substrato se demonstrou eficiente para a colonização do mexilhão dourado em programas de 
monitoramento desta espécie invasora na área do delta do rio Jacuí.

\section{Agradecimentos}

A equipe de campo do Laboratório de Malacologia pelo apoio logístico. Ao CNPq/CT-Hidro (processo $\mathrm{n}$. 507675/2004-5) pelo auxílio concedido.

\section{Referências}

Boltovskoy, D.; Cataldo, D. 1999. Population dynamics of Limnoperna fortunei, an invasive fouling mollusc, in the Lower Paraná River (Argentina). Biofouling, 14 (3): 255-263.

Bendati, M. M.; Schwarzbach, M. S. R.; Maizonave, C. R. M.; Almeida, L. B.; Bringhenti, M. L. 2000. Avaliação da Qualidade da água do lago Guaíba (Rio Grande do Sul, Brasil) como suporte para a gestão da bacia hidrográfica. Anais do XXVII Congresso Interamericano de Engenharia Sanitária e Ambiental, Porto Alegre, Brasil, p.1-20.

Conselho Nacional do Meio Ambiente (CONAMA). Resolução no 20, Ministério do Desenvolvimento Urbano e Meio Ambiente. Diário Oficial da União, 30 de julho de 1986.

Darrigran, G. E.; Mansur, M. C. D. 2006. Distribuição, abundância e dispersão. In: Darrigran, G. A. \& Damborenea, C. (Eds). Bio-invasion del mejillón dourado en el continente americano. Edulp, La Plata, Argentina, p.93-110.

Darrigran, G. E.; Damborenea, C.; Greco, N. 2007. Freshwater invasive bivalves in man-made environments: A case study of larvae biology of Limnoperna fortunei in a Hydroelectric Power Plant in South America. AMBIO, 36 (7): 575- 579.

Faria, C. M.; Lersch, E. C. 1998. Delta do Jacuí: Águas e sedimento. DMAE, Porto Alegre, Brasil, 56pp.

Fontes, H. M.; Takeda, A. M.; Fugita, D. S. 2002. Experience in Itaipu reservoir (Brazil) with artificial substrate (in situ): is it best way of monitoring an invading species like Limnoperna fortunei (Bivalvia, Mytilidae)? Proceedings of International Symposium on Reservoir Management in Tropical and Sub-Tropical Regions, Foz do Iguaçu, Brasil, p.673-679.

Mansur, M. C. D. 2007. O mexilhão dourado Limnoperna fortunei (dunker, 1857) invasor de origem asiática no Sul do Brasil (Bivalvia, Mytiloida, Mytilidae). Tópicos em Malacologia - Ecos do XVIII EBRAM, Rio de Janeiro, Brasil, p.71-80.

Mansur, M. C. D.; Cardoso, F. R.; Ribeiro, L. A.; Santos, C. P.; Thormann, B. M.; Fernandes, F. C.; Richinitti, L. M. Z. 2004. Distribuição e conseqüências após cinco anos da invasão do mexilhão dourado, Limnoperna fortunei, no estado do Rio Grande do Sul, Brasil (Mollusca, Bivalvia, Mytilidae). Biociências, 12 (2): 165172.
Mansur, M. C. D.; Pereira, D. 2006. Bivalves límnicos da bacia do rio dos Sinos, Rio Grande do Sul, Brasil (Bivalvia, Unionoida, Veneroida e Mytiloida). Revista Brasileira de Zoologia, 23 (4): 1123-1147.

Mansur, M. C. D.; Richinitti, L. M. Z.; Santos, C. P. 1999. Limnoperna fortunei (Dunker, 1857), molusco bivalve invasor, na bacia do Guaíba, RS, Brasil. Biociências, 7 (2):147-150.

Mansur, M. C. D.; Santos, C. P.; Darrigran, G.; Heydrich, I.; Callil, C. T.; Cardoso, F. R. 2003. Primeiros dados quali-quantitativos do mexilhão dourado, Limnoperna fortunei (Dunker), no Delta do Jacuí, no Lago Guaíba e na Laguna dos Patos, Rio Grande do Sul, Brasil e alguns aspectos de sua invasão no ambiente. Revista Brasileira de Zoologia, 20 (1): 75-84.

Marques-Silva, N. S.; Beasley, C. R.; Gomes, C. P.; Gardunho, D. C. L.; Tagliaro, C. H.; Schories, D.; Mehlig, U. 2006. Settlement dynamics of the encrusting epibenthic macrofauna in two creeks of the Caeté Mangrove Estuary (North Brazil). Wetlands Ecology and Management, 14 (1): 67-78.

Morton, B. 1982. The population dynamics of Limnoperna fortunei (Dunker, 1857) (Bivalvia: Mytilacea) in Plover Cove Reservoir, Hong Kong. Malacologia, 16 (1): 165-182.

Oliveira, M. D.; Takeda, A. M.; Barros, L. F.; Barbosa, D. S.; Resende, E. 2006. Invasion by Limnoperna fortunei (Dunker, 1857) (Bivalvia, Mytilidae) of Pantanal wetland, Brasil. Biological Invasions, 8: 97-104.

Santos, C. P. 2004. Avaliação da densidade e crescimento populacional do mexilhão dourado Limnoperna fortunei (Dunker, 1857) em suas diferentes fases de vida no lago Guaíba, município de Porto Alegre, RS, como subsídios ao controle do bivalve invasor. Dissertação de Mestrado, Universidade Federal do Rio Grande do Sul, Brasil, 93pp.

Santos, C. P.; Würdig, N. L.; Mansur, M. C. D. 2005. Fases larvais do mexilhão dourado Limnoperna fortunei (Dunker) (Mollusca, Bivalvia, Mytilidae), na bacia do lago Guaíba, Rio Grande do Sul, Brasil. Revista Brasileira de Zoologia, 22 (3): 702-708.

Santos, C. P.; Würdig, N. L.; Mansur, M. C. D. 2008. Variações no comprimento dos indivíduos de uma população do mexilhão dourado, Limnoperna fortunei (Mollusca: Bivalvia: Mytilidae), ao longo do ano, na Praia do Veludo, lago Guaíba, Rio Grande do Sul, Brasil. Revista Brasileira de Zoologia, 25 (3): 389-396.

Terra, N. R.; Lemieszek, M. B.; Lemos, C. T.; Leite, E. H. 2007. Presença de Limnoperna fortunei (Dunker, 1857) nas bacias hidrográficas do Uruguai e lago Guaíba, Rio Grande do Sul, Brasil. FEPAM em Revista, 1 (1): 12-19.

Zar, J. H. 1999. Biostatistical Analysis. Prentice Hall, New Jersey, USA, 662pp. 